\title{
The representational basis of syllable categories
}

\author{
STEF DECOENE \\ Katholieke Universiteit Leuven, Leuven, Belgium
}

\begin{abstract}
Two hypotheses concerning the relation between phoneme and syllable categories are contrasted. The phonological hypothesis holds that phoneme categories are perceptually basic, whereas syllable categories are abstract and specify the phonological rules operating on phoneme categories. The integrative routine hypothesis suggests that whereas phoneme categories are basic, syllable categories are not entirely abstract but are represented at least partially on the basis of higher order perceptual information, specifying the dynamics of coarticulating phoneme categories. Three experiments used a primed naming task in which subjects had to name the entire syllable (consonant-vowel and vowel-consonant; Experiment 1), the syllable-initial phoneme category (Experiment 2), or the syllable-final phoneme category (Experiment 3). The results supported the integrative routine hypothesis.
\end{abstract}

Although the role of phoneme and syllable categories during speech perception and lexical processing has been studied extensively, and although much research has been directed at what information constitutes phoneme categories (see Pisoni \& Luce, 1987, for a review), little is known about the representational basis of syllable categories. In the research reported here, two hypotheses concerning the content of these syllable representations and their relation with phoneme representations are constrasted. On one interpretation, syllable representations could consist of abstract (nonperceptual) information. A likely format would be a phonological structure with some sort of a combination rule operating on a sequence of phoneme categories (henceforth the phonological hypothesis). Another interesting possibility, however, is that syllable categories are (at least partly) represented by higher order perceptual information describing how phoneme categories are combined during speech production. In this case, they would specify the articulatory dynamics of concatenating phoneme categories (the integrative routine hypothesis; see Browman \& Goldstein, 1986, 1988, 1989, 1990; Sussman, 1984, 1986, 1989; Whalen, 1989, for comparable suggestions). Both hypotheses assume that phoneme categories are perceptually basic, but differ with respect to the exact representational content of syllable categories.

The distinction between the two hypotheses should not be reduced to the question of whether coarticulatory cues are used during speech perception. Coarticulatory infor-

S.D. is a postdoctoral fellow of the Belgian Fund for Scientific Research. The research reported here is part of his doctoral dissertation, The perceptual representation of phoneme and syllable categories (supervisor: G. Govaerts). The author is grateful to Terry Nearey, John Kingston, and Jo Baele for their help in improving the manuscript, to Rob Stroobants for advice on data analysis, and to J. Kerstens (Intelligent Computers) and Chris Caerts (ESAT) for technical assistance. Support from a National Fund of Scientific Research grant (S2/5-ID.E120) is gratefully acknowledged. Correspondence should be addressed to S. Decoene, Diestsesteenweg 242, 3010 Kessel-Lo, Belgium (e-mail: stef.decoene@psy.kuleuven.ac.be). mation is clearly used during phoneme perception (see Strange, 1989, and Handel, 1989, for reviews). The phonological hypothesis and the integrative routine hypothesis differ with respect to the question of whether coarticulatory cues form, at least partly, the representational basis of syllable categories. According to the former hypothesis, syllable representations are not based on coarticulatory cues, whereas according to the latter they do.

Several lines of research could in principle be used to illuminate the representational basis of syllable categories. One indication that syllable structure plays a role in speech perception is that when subjects are asked to identify the vowels in sequences of rapidly repeated syllables, they perform much better when formant transitions are included than when these transitions are removed, because formant transition preserves the perceptual integrity of the syllables (Cole \& Scott, 1973, 1974; Dorman, Cutting, \& Raphaël, 1975).

Ades (1974) reported that selective adaptation effects on phoneme category identification were position dependent. Adapting with a [b]V syllable produced an effect on the identification of [b] in [b] V but not in V[b] syllables. This phenomenon was replicated by Pisoni and Tash (1975), Sawusch (1977), and Wolf (1978), and extended to intervocalic consonants (Samuel, Kat, \& Tartter, 1984). Pisoni and Tash (1975) and Sawusch (1977) also manipulated formant transitions of consonant-vowel (CV) and vowel-consonant (VC) syllables and showed that some adaptation effect could be obtained if, for example, CV syllables were made more like VC syllables. Such a manipulation suggests that this phenomenon is related to coarticulatory information, and thus to the acoustically specified syllable structure. Samuel et al. (1984; see also Samuel, 1989) concluded explicitly that the positiondependent effect of selective adaptation shows that the syllable is an important perceptual unit.

Another line of research that could speak to the issue of speech representations concerns the presence or absence of context effects during speech perception, in particular the way in which phonological information influ- 
ences perceptual information. For example, Massaro and Cohen (1983) showed that identification of the glides $/ \mathrm{r} /$ and $/ 1 /$ was influenced by whether the preceding context was phonologically admissible or not. They modeled the data using different frameworks and argued in favor of a model that assumes that perceptual and phonological information are independent.

However, these results and their modeling by, for example, Massaro and Cohen (1991) and McClelland (1991) much more address processing than they do perceptual/ cognitive representation. Massaro and Cohen's fuzzy logical model of perception (FLMP) assumes syllablesized prototype representations at which the independent sources of perceptual and phonological information are integrated (see also Massaro, 1987, 1989). McClelland's (1991; see also Elman \& McClelland, 1986) (stochastic) interactive activation and competition (SIAC) and TRACE models assume phoneme representations at which featural and phonological information are interactively combined. These models speak to the issue of speech representation only insofar as they assume a specific representation at which information sources come together. For example, whereas the SIAC model has as its representational units features, phonemes, and words, it would function equivalently if its units were features, phonemes, and syllables. In that case, the SIAC model would offer one implementation of the phonological hypothesis with respect to syllable representations: Phoneme representations have a perceptual content (stimulus features map onto phoneme units), whereas syllable representations are phonologically specified (phoneme units, but not features, map onto syllable units). But again, it could equally well instantiate the integrative routine hypothesis if direct connections between syllabic units and certain stimulus features were included, besides connections between certain stimulus features and phoneme units.

Nevertheless, research on the interaction between information sources exists within the specific context of research on the speech unit hypothesis. Whalen (1989) investigated whether consonant and vowel information (in this case, duration of the vocalic segment) in [/b/]VC syllables interacted when subjects had to identify the syllable. Important is that via speech synthesis vowel duration can be made to specify jointly both the $[\mathrm{d}] /[\mathrm{t}]$ and the ae/e distinction. His results supported the idea that vowel and consonant judgments are not independent. Whalen noted that his results could in principle be evidence for the syllable as the primary perceptual unit, but that this was far from a necessary conclusion. Another possible interpretation is that syllables constrain their constituent phoneme perception through "a mapping from segments onto the syllable's acoustic realization - the fact that the same information is being used for both signifies that both judgments must make sense in relation to each other as well as to the input itself" (Whalen, 1989, p. 291).

The representational implications of context effects in speech perception have been taken up directly by Nearey
(1990; see also Nearey, 1991, 1992), who has presented logistic analyses of the results of Massaro and Cohen (1983), and Whalen (1989). Nearey showed that context effects could be most parsimoniously explained by a diphonebiased segmental model. This model has a multilayered architecture in which "the first layer comprises a set of segmental filters wherein all stimulus tuning takes place, while higher-level units implement additive, stimulusindependent corrections for (passive) coarticulation, (preplanned) extrinsic allophony and phonotactic constraints" (Nearey, 1991, p. 47). In terms of speech representations this implies that (1) phoneme categories are represented on the basis of perceptual information resulting in contextsensitive units, whereas (2) higher order representations, such as syllables, are not perceptually defined, even if they partly reflect, for example, coarticulation. With respect to the issue addressed here, Nearey's model is an implementation of the phonological hypothesis concerning syllable representations-syllable representations are represented, but their representational content is not perceptually defined.

Finally, Decoene (1993; Decoene \& Timperman, 1996) used a primed-phoneme and syllable-matching task under variable matching instructions to test directly the degree to which phoneme categories, syllable categories, or both were represented on the basis of perceptual information. The results from the primed phoneme matching experiments clearly indicated that the representational content of phoneme categories consists of perceptual information only. The primed syllable-matching experiments did not support such a direct perceptual determination of syllable categories. Consequently, Decoene (1993) concluded that phoneme categories are the basic speech units. However, although the primed syllable-matching results could be used as support for the phonological hypothesis, Decoene (1993) also found some evidence that primed syllable matching under same-name instructions was at least partially based on perceptual information, which better fits the integrative routine hypothesis. Decoene (1993) concluded that although phoneme categories are perceptually basic, syllable categories could possibly be based on higher order perceptual information that specifies how their constituent phoneme categories are coarticulated. As such, the data of Decoene (1993) do not fit with the representational assumptions of the FLMP, TRACE, and the diphone-biased segmental model. FLMP has perceptually defined syllable representations but no phoneme representations. TRACE (and SIAC) have perceptually defined phoneme representations but no syllable representations. And Nearey's (1990) diphone-biased segmental model has perceptually defined phoneme representations and phonologically specified syllable representations.

Research is necessary to shed more light on the representational basis of syllable categories. Here, three experiments contrast the phonological hypothesis with the integrative routine hypothesis. Both hypotheses assume that phoneme categories are represented on the basis of 
perceptual information and that syllable categories are represented, but the hypotheses differ in terms of how syllable representations are defined.

\section{EXPERIMENT 1 Primed Syllable Identification}

Suppose that subjects have to name, as fast as possible, auditorily presented $\mathrm{CV}$ and VC syllables and that they are helped by previously presented information. Using such a priming paradigm forces subjects to focus on the representational aspects of the task they are confronted with. This is needed to address the explicitly representational issue studied here. Priming activates a representation insofar as the priming stimulus resembles the primed stimulus in some way. Therefore, any effect of priming should be attributed to facilitation at the representational level, not at the level of processing demands or functions. Using a response time (RT) measure offers an additional guarantee and control for this representational focus.

Given CV and VC syllables, three kinds of priming information can be presented: the orthographically presented syllable category name ( $\mathrm{S}$ priming), the syllable-initial phoneme category (IP priming), or the syllable-final phoneme category (FP priming). IP priming and FP priming will activate the perceptually defined representation of, respectively, the syllable-initial or the syllablefinal phoneme category (Information Sources 1 and 2). $S$ priming will activate both its perceptually defined phoneme category representations and the syllable representation (Information Source 3) - phonologically specified in case the phonological hypothesis is correct, or specified by higher order perceptual information in case the integrative routine hypothesis is correct.

Under the phonological hypothesis, syllable identification will depend on the speed with which the syllablefinal phoneme is perceived, because both the phonological syllable structure and the acoustic signal are defined from left to right: phonologically and acoustically first onset, then nucleus, and finally coda. From this it follows that syllable identification can be made only after all phoneme categories have been perceived. Therefore, response facilitation by $\mathrm{S}$ priming can be expected only through Information Source 2. FP priming will facilitate responses in the same way as $\mathrm{S}$ priming because the locus of the effect is the same (Information Source 2). However, no such facilitation should be expected if subjects receive IP priming. Although IP priming will activate the perceptually defined syllable-initial phoneme category, the syllable representation cannot be found before the syllable-final phoneme has been perceived. Therefore, IP priming should be equivalent to a no priming condition. One might object that subjects can start guessing which syllable is presented if they already know the initial phoneme category. However, in the case of $\mathrm{CV}$ and VC syllables, most combinations between vowels and consonants are acceptable (in Dutch), so guessing strategies would result in a lot of mistakes.
Given the integrative routine hypothesis, S priming will activate higher order perceptual information defining the syllable (Information Source 3), plus perceptual information about the two constituent phoneme categories (Information Sources 1 and 2). IP priming will activate perceptual information facilitating perception of the syllable-initial phoneme category. However, when subjects start hearing the auditorily presented syllable, this should result not only in an earlier perception of this syllable-initial phoneme category, but indirectly also in an easier detection of the way in which the actual perceptual specification of this phoneme is modified by the dynamic information of coarticulating the syllable-initial and syllable-final phoneme category. That is, the higher order perceptual information will be easier to detect precisely because it is dependent on the perceptual information specific to the syllable-initial phoneme category. Of course, $S$ priming will have a larger effect than IP priming because Information Sources 1 and 3 will be jointly and directly activated. For FP priming, the prediction is more difficult to make. Some facilitation, however, could be expected. The higher order perceptual information present in the acoustic signal will help to specify which phoneme categories have been coarticulated, and the perceptual information activated through FP priming will strengthen this. Possibly this effect could be smaller than for IP priming, given the tradeoff between the left-to-right presentation of the stimulus syllables and the locus of the priming stimulus.

In summary, the consequences for RTs of the phonological hypothesis for primed syllable identification are $\mathrm{S}$ priming $\mathrm{RT}=$ FP-priming $\mathrm{RT}<\mathrm{IP}$-priming $\mathrm{RT}=$ control $\mathrm{RT}$, and for the integrative routine hypothesis: S-priming $\mathrm{RT}<\mathrm{IP}$-priming RT $\leq$ FP-priming RT < control RT.

It should be noted that these predictions depend on how the perceptual information defining the phoneme category is understood. In Decoene (1993; Decoene \& Timperman, 1996), prototype representations were assumed, indicating how "ideal" phonemes should sound. Consequently, phoneme categories are most easily understood as based on stable, context-independent information. However, graded structure could also be generated by category representations based on, for example, exemplars or on mixed prototype-plus-exemplar structures (see, e.g., Medin \& Barsalou, 1987). In that case, phoneme categories would most likely be context-dependent representations, and for each phoneme constitute, for example, the range of allophonic variation. Here I will take the prototype view, used in Decoene (1993), for granted. I will return to this issue in the discussion.

Also, perception and identification are explicitly distinguished in making the predictions (see Foss \& Swinney, 1973). Syllable identification is not necessarily dependent on phoneme identification (which implies conscious access to a representation), but only on phoneme perception (the activation of a perceptual representation). In fact, it follows from the phonological hypothesis that syllables are strictly speaking not perceived, but constructed out of perceived phoneme representations. 


\section{Method}

Stimuli. The stimuli were constructed using the following consonants and vowels. As far as consonants are concerned, two stops (voiced/unvoiced) were included: /p/,/b/; one liquid, $/ \mathrm{l} ;$; a fricative, $/ \mathrm{s} / ;$ a glide, /w/; and a nasal, $/ \mathrm{n} /$. The vowels chosen were $/ \mathrm{a} /, / \mathrm{a}: /$, $/ 0 /, / 0: /, / y /$, and $/ \mathrm{u} /$, representing a fair sample from different points in vowel space (see, e.g., Clark \& Yallop, 1990; Collier \& Droste, 1982), and having an unambiguous relation between grapheme and phoneme. The vowels and consonants were combined into two syllable types, $\mathrm{CV}$ and VC, resulting in 72 syllables. It should be noted that 11 of the 72 stimuli were also words $(/ \mathrm{pa} /, / \mathrm{a}: \mathrm{p} /, / \mathrm{op} /, / \mathrm{al} /, / \mathrm{a}: \mathrm{l} /$, /as/, /a:s/, /os/,/na/,/nu/, and /a:n/) — controlling the individual data points in the three experiments for these 11 stimuli did not show any outliers. The stimuli were recorded by a female speaker in a soundproof room with a Stellavox SP7 recorder and Sennheiser MD441N microphone. The speaker was instructed not to devoice the VC syllables ending with $/ \mathrm{b} /$ in order to keep the contrast between $\mathrm{V} / \mathrm{b} /$ and $\mathrm{V} / \mathrm{p} /{ }^{1}$ Afterward, the speaker and the experimenter listened to the recording. When either the speaker or the experimenter felt unsure about the quality of a pronounced syllable, it was recorded again. The syllables were 16 bit digitalized at $32 \mathrm{kHz}$ using the FFAD021650K A/D convertor and stored on hard disk.

For each spoken syllable stimulus, four priming conditions were included: a syllable prime (e.g., $A P$ ), an initial phoneme prime (e.g., $\left.A_{-}\right)$, a final phoneme prime (e.g., $\_P$ ), and a control condition ( () . Twenty percent of catch trials were included to avoid response strategies by subjects. As shown by Posner and Snyder (1975), a ratio of $80 \%$ correct and $20 \%$ wrong primes allows maximal facilitation by priming, combined with small inhibition on catch trials. A catch trial could take three forms: a wrong-syllable prime (e.g., $P A-\left[\right.$ ny]), a wrong initial-phoneme prime (e.g., $P_{-}-$ [ny]), or a wrong final-phoneme prime (e.g., $A-[$ ny]). All in all, the experiment consisted of 360 stimulus trials. Each syllable type (72) was preceded by the four priming conditions, giving 288 stimulus trials and 72 catch trials.

Procedure. The Experiment was computer controlled, using a parallel Pascal program running on a T800 transputer with a PC/AT as host. A voice key was connected to the PC/AT. The spoken syllables were presented via the FFDA021650K D/A convertor connected to a Philips sound amplifier. Subjects listened to the spoken syllables over an MB H805 headphone. Instructions were presented via a monochrome monitor. Subjects were told that they had to pronounce the entire auditorily presented syllable. The possible primes were described, and two (visually presented) examples were given. Subjects were asked to respond as fast as possible, without making too many mistakes. Stress was put on fast responses. They were asked to mentally rehearse the visually presented prime after reading it. Subjects were warned that catch trials were included.

At the beginning of the experiment, stimuli were randomized a first time. After each block of 50 stimuli, the remaining set of stimuli was randomized again. Initial seeds were varied over subjects. Each stimulus trial consisted of a visually presented prime (in orthographic form) followed by an auditorily presented syllable. The interval between prime and spoken syllable could not be controlled exactly, because it depended in part on the length of the digitalized syllable file. This was due to an interplay between software and hardware limitations. The program that controlled the experiments was written in such a way that this limiting factor was reduced to a minimum. Moreover, the digitalized stimulus files were standardized with respect to length as much as possible. The interval varied between 1,600 and 1,700 msec.

When the subjects had identified the syllable category, the correctness of their answers was assessed by the experimenter and input to the PC/AT. As soon as the correct/wrong information was passed to the computer, the following stimulus trial was presented. Subjects were given the opportunity to pause after each block of 50 stimuli. After each block they received some feedback (average RT and number of errors) for all presented stimuli. If subjects made a mistake, the stimulus trial was presented again later during the experiment. In this way the amount of missing data could be reduced to a minimum.

Subjects. Thirty-one subjects participated in partial fulfillment of course credit. None reported any hearing deficiencies. Subjects who made more than $10 \%$ errors overall or more than $15 \%$ errors on catch trials were excluded. After scrutinizing the data, 2 subjects were removed: 1 subject was not a native speaker, and the other made more than $15 \%$ errors on catch trials.

\section{Results and Discussion}

The following data points were considered missing: RTs higher than $2,000 \mathrm{msec}$ and stimuli responded wrongly to more than three times. When use of the voice key occasionally resulted in a value of close to zero, this too was defined as missing. Using these criteria, 40 data points were defined as missing. Data points were then averaged over stimulus instances, yielding a randomized factorial block model (Kirk, 1982) with syllable type, priming, and its two-way interaction as effects. Since the hypotheses are detailed and specified a priori, Bonferroni tests $(p=.05)$ were computed where allowed.

$S$ priming facilitated syllable identification most ( $533 \mathrm{msec})$, followed by FP priming $(578 \mathrm{msec})$ and IP priming ( $581 \mathrm{msec}$ ), which did not differ, and the control $(612 \mathrm{msec})$. This overall effect of priming was significant $[F(3,196)=79.75, p \leq .0001$. A priori Bonferroni tests $\left[t(196)=2.64, M S_{\mathrm{e}}=784.2\right]$ showed that the observed ordering of the RTs for S-priming RT < FP-priming $\mathrm{RT}=\mathrm{IP}$-priming RT < control RT was significant. Therefore, the integrative routine hypothesis is supported.

$\mathrm{CV}$ syllables were responded to significantly more slowly than were VC syllables, 610 versus $542 \mathrm{msec}$, $[F(1,196)=343.43, p \leq .0001]$. An interpretation on the basis of differences in coarticulatory coding between VC and CV syllables (e.g., Pols, 1977), with VC syllables more strongly coded than CV syllables, could be put forward to explain this effect.

\section{EXPERIMENT 2 \\ Primed Syllable-Initial Phoneme Identification}

The results of Experiment 1 confirmed the integrative routine hypothesis. However, this hypothesis would be supported even more strongly if it could be shown that it also correctly predicts naming of the phonemes constituting the syllable. For this reason, a second experiment was run in which subjects were asked to name the initial phoneme of auditorily presented syllables under the same priming conditions as those in Experiment 1. Applying the same logic as that from Experiment 1, one can predict that syllable-initial phoneme identification under the phonological hypothesis should yield the following pattern: S-priming RT = IP-priming RT < FP-priming $\mathrm{RT}=$ control RT. Given this hypothesis, the only effective piece of information activated by $S$ priming will be perceptual information specifying the initial phoneme category, making $\mathrm{S}$ priming functionally equivalent to IP priming. 
Given the integrative routine hypothesis, one should predict: S-priming RT < IP-priming RT < FP-priming RT $=$ control RT. S priming and IP priming differ because under this hypothesis, $S$ priming will activate coarticulatory information associated with the syllable representation, in addition to perceptual information associated with the initial phoneme category representation. Given the prototype view of phoneme category representation, FP priming should have no effect, because priming activates the context-independent perceptual description of the final phoneme, which will not aid syllable-initial phoneme identification.

\section{Method}

Stimuli, Experimental Design, and Procedure. The same stimuli, experimental design, and procedure as those in Experiment 1 were used. The only exception was that subjects were asked to name as fast as possible the syllable-initial phoneme of the auditorily presented syllables. They were explicitly told that naming an entire syllable would be considered an error.

Subjects. Thirty-two subjects participated in partial fulfillment of course credit. None of the subjects had participated in Experiment 1 . None reported any hearing deficiencies. After selection, 29 subjects remained: 2 subjects had more than $10 \%$ errors overall and more than $15 \%$ errors on catch trials, and the $3 \mathrm{rd}$ had more than $15 \%$ errors on catch trials

\section{Results and Discussion}

Fifty data points were excluded from the data set using the criteria explained for Experiment 1. The data were analyzed as in Experiment 1.

Naming was facilitated more by S priming $(541 \mathrm{msec})$ than by IP priming $(577 \mathrm{msec})$. Both facilitated more than FP priming ( $644 \mathrm{msec}$ ) and no-priming ( $655 \mathrm{msec}$ ), which in turn did not markedly differ. This main effect of priming was significant $[F(3,196)=106.76, p \leq .0001]$. A priori Bonferroni tests confirmed this ordering $[t(196)=$ 2.64, $\left.M S_{\mathrm{e}}=1,624.7\right]$. Again, the differential effect of various priming conditions on syllable-initial phoneme naming ( $\mathrm{S}$ priming $>\mathrm{IP}$ priming $>\mathrm{FP}$ priming $=$ no priming) supports the predictions made by the integrative routine hypothesis.

VC syllables were responded to faster than $\mathrm{CV}$ syllables, 598 versus $610 \mathrm{msec}[F(1,196)=5.53, p<.05]$. As for Experiment 1, a likely explanation can be given on the basis of differences in coarticulatory coding between VC and CV syllables (see, e.g., Pols, 1977).

\section{EXPERIMENT 3 \\ Primed Syllable-Final Phoneme Identification}

The results of Experiment 2 further corroborated the integrative routine hypothesis. To complete the testing of the predictions derived from this hypothesis, in Experiment 3 subjects were asked to identify the syllablefinal phoneme of auditorily presented syllables. Applying the logic explained in Experiment 1, predictions for the phonological hypothesis are as follows: S-priming $\mathrm{RT}=$ FP-priming $\mathrm{RT}<\mathrm{IP}$-priming $\mathrm{RT}=$ control. Given the integrative routine hypothesis, the following pattern of results is expected: S-priming RT $<$ FP-priming $\mathrm{RT}<$ IP-priming RT $<$ control RT.

\section{Method}

Stimuli, Experimental Design, and Procedure. Stimuli, experimental design, and procedure were identical to those of Experiments 1 and 2, the only exception being that subjects were asked to name as fast as possible the syllable-final phoneme of the auditorily presented syllables.

Subjects. Thirty-three subjects were run in partial fulfillment of course credit. None of the subjects had participated in Experiment 1 or 2. None reported any hearing deficiencies. After selection, 29 subjects remained: One subject proved to be ill, and the others had more than $15 \%$ errors on catch trials.

\section{Results and Discussion}

Using the criteria described for Experiment 1, 33 data points were excluded from the data set. The remaining data were analyzed as in Experiments 1 and 2.

S priming facilitated RTs most $(665 \mathrm{msec})$, followed by FP priming ( $703 \mathrm{msec})$, IP priming $(764 \mathrm{msec})$, and the control condition $(787 \mathrm{msec})$. The overall effect of priming was significant $[F(3,196)=107.91, p \leq .0001]$. A priori Bonferroni tests showed that all four levels differed significantly from one another $\left[t(196)=2.64, M S_{\mathrm{e}}=\right.$ $1,645.2]$. Clearly, this pattern again conforms to the predictions made by the integrative routine hypothesis.

However, the type $\times$ priming interaction was also significant $[F(3,196)=3.88, p=.01]$ (Figure 1). A posteriori Tukey tests $(p=.05)$ were computed. Within CV syllables, the effect of priming was significantly different among all levels in the order predicted by the integrative routine hypothesis: S-priming RT $(682 \mathrm{msec})<$ FPpriming RT (704 msec) < IP-priming RT (757 msec) < control $(798 \mathrm{msec})$. Within VC syllables, however, the difference between IP priming and the control was not significant: S-priming RT $(649 \mathrm{msec})<$ FP-priming

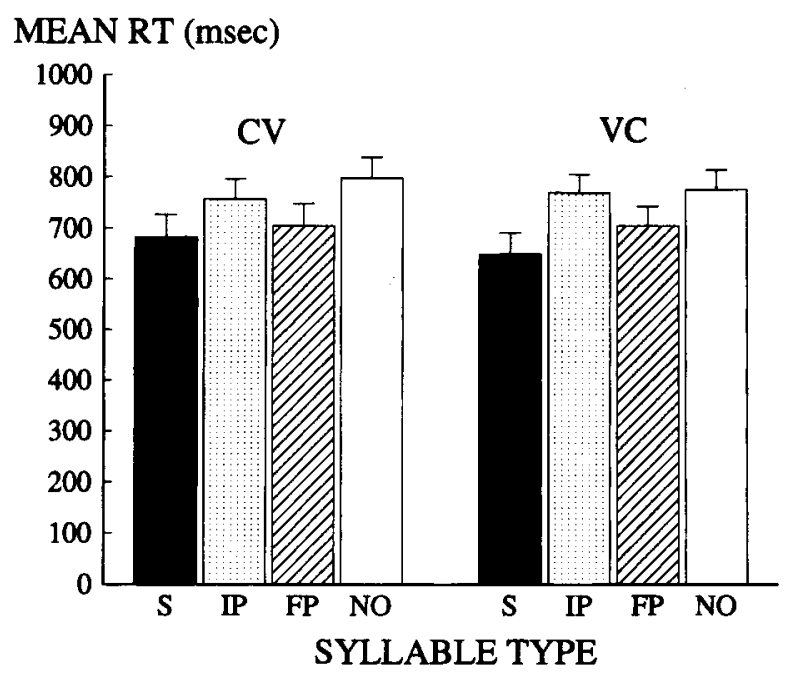

Figure 1. The results of Experiment 3 (syllable-final phoneme identification) for consonant-vowel ( $\mathrm{CV}$ ) and vowel-consonant (VC) syllables separately. S, S-priming condition; IP, IP-priming condition; FP, FP-priming condition; NO, control condition. 
$(705 \mathrm{msec})<$ IP-priming RT $(769 \mathrm{msec})=$ control $(776 \mathrm{msec})$. Moreover, S priming affected VC syllables significantly more than $\mathrm{CV}$ syllables, and naming in the control condition was faster for VC syllables than for CV syllables.

According to this interaction, then, the integrative hypothesis is supported with CV syllables, but only partially for VC syllables. However, the discrepancy is, I think, a minor one. There are two reasons for this. First, the results of the three experiments overwhelmingly support the integrative routine hypothesis. Second, and more importantly, if one accepts that $\mathrm{VC}$ syllables are indeed more strongly coded in coarticulatory terms (Pols, 1977), and that the integrative routine hypothesis is correct, then the priming pattern for $\mathrm{VC}$ syllables can be explained, not by reference to IP priming, but by reference to the control and S-priming conditions. Given strong coarticulatory coding of VC syllables, one would expect that VC syllables are named more rapidly than CV syllables in the control condition because more information is available for these VC syllables, and more rapidly in the S-priming condition because this more strongly coded information is primed through the activation of syllable representations. In that case, syllable-final phoneme identification can be aided by IP priming because this kind of priming helps recovery of coarticulation effects, but it becomes quite possible that the benefit of IP priming is simply too small relative to the no-priming condition, given strong coarticulatory coding of VC syllables.

\section{GENERAL DISCUSSION}

The results of the three experiments support the integrative routine hypothesis. In this view, the representational architecture of speech perception looks something like this. On the first representational layer are phoneme category representations that are activated on the basis of perceptual information. The perceptual content of these phoneme categories refers to stable (context-free) perceptual information and is organized by prototypes. On the second layer are syllable category representations that are at least partly based on higher order perceptual information specific to the acoustic consequences of coarticulatory dynamics. It is important to stress that syllable category representations are not purely perceptually defined but that their representational content also seems to be at least lexically relevant (Decoene, 1993; Decoene \& Timperman, 1996). A straightforward description could be that syllable representations are partly perceptually and partly phonologically defined, and thus function as intermediary between purely perceptually defined phoneme categories and lexical units.

The results are consonant with Massaro's FLMP in the sense that there are (at least partially) perceptually determined syllable category representations, but not in the sense that syllable category representations are built on perceptually determined phoneme category representations-which in the FLMP are not even represented. A version of FLMP could be developed by incorporating in it an extra layer of phoneme-sized prototypes. Although this may seem a rather drastic measure, it should be recognized that, as far as I know, experiments using the FLMP as a framework for research on speech perception use perceptual features that often more readily describe phoneme categories than syllable categories per se (see, e.g., the description of stimuli in Massaro \& Cohen, 1983). In this light, the feature integration stage of the FLMP could be considered to imply phoneme category representations "in disguise."

The results are compatible with variants of TRACE insofar as it holds that phoneme categories are represented on the basis of perceptual information, but are incompatible with its assumption that syllable categories are phonological. However, TRACE-like models can easily deal with the representational view taken here by allowing connections between syllabic units and at least some perceptual features.

Both TRACE and FLMP can be changed with respect to their representational assumptions without affecting their respective processing structure. This is not the case for Nearey's diphone-biased segmental model, in which the representational architecture is its crucial element. Therefore, it is worthwhile to analyze in more detail the degree to which it can predict the results of the three experiments reported here. In spelling out these predictions, there are two crucial elements. First of all, activation of syllable representations has no perceptual effects, because their representational content is abstract/phonological. Second, the content of phoneme categories is context sensitive. This implies that, for example, activation of a syllable-initial phoneme category of a CV syllable will also result in the reduction of the set of neighboring segment candidates, thus facilitating the perception of the syllable-final phoneme category without the necessarily prior activation of, for example, the syllable category representation.

For the syllable-naming experiment, taking this into account yields the following: S-priming RT $<$ IP-priming $\mathrm{RT}=$ FP-priming RT < control RT (observed ordering: S-priming $\mathrm{RT}<\mathrm{IP}$-priming $\mathrm{RT}=\mathrm{FP}$-priming $\mathrm{RT}<$ control RT); for the syllable-initial phoneme-naming experiment: S-priming RT < IP-priming RT < FP-priming RT < control RT (observed ordering: S-priming RT < IP-priming RT < FP-priming RT = control RT); and for the syllable-final phoneme-naming experiment: S-priming RT < FP-priming RT = IP-priming RT < control RT (observed ordering: S-priming RT $<$ FPpriming RT $<$ IP-priming RT $<$ control RT). There are two deviations of the predicted from the observed patterns. In the case of syllable-initial phoneme naming, FP priming should facilitate RTs with respect to the control condition, because in a diphone-biased segmental model with context-sensitive phones, any priming should facilitate responses-specifically, FP priming also offers information about the syllable-initial phoneme category. In the case of syllable-final phoneme naming, one would expect equivalent facilitation of IP priming and FP priming. FP priming will unambiguously activate the syllable- 
final phoneme category so that subjects will be able to respond soon after this phoneme is presented, whereas IP priming will reduce the neighboring segment candidates so that, again, subjects will be able to respond soon after the syllable-final phoneme is presented. This prediction is based on the combination of facilitation by priming and of the necessarily left-to-right nature of the perceptual presentation of the stimuli. In sum, a diphonebiased segmental model, although making better predictions than the phonological hypothesis, does worse than the integrative routine hypothesis, which holds that syllable representations are at least partially based on perceptual information.

However, T. M. Nearey (personal communication, May 25, 1996) has pointed out that new diphone-biased segmental models implementing the phonological hypothesis can be constructed that predict the reported results better than the model discussed in the previous paragraphs. If a model of the type sketched by Nearey (1990, pp. 369-370) is modified so that choices made are based on the average activation level of phoneme-level units and of all syllable representations compatible with the activated phoneme representations, this version of the phonological hypothesis predicts the results obtained in Experiment 3, although still not those in Experiment 2.

But, a problem with this ad hoc assumption is that its perceptual function is unclear. The functional advantage of such a recoupling of syllabic activations into the activation of phoneme representations would make sense if it would improve the probability of correct phoneme representation. Since in Dutch most phonological CV and VC combinations are acceptable (with the exception of VC syllables ending with $/ \mathrm{b} /, / \mathrm{d} /, / \mathrm{v} /$, and $/ \mathrm{z} /$ ), such a recoupling would not differentiate which syllable was more probable than any other given the stimulus presented. Although such a differentiation is likely when the activation of syllable representation is under at least partial control of the perceptual features of the stimulusthat is, when a version of the integrative routine hypothesis is correct-it is unclear how it could be achieved given some version of the phonological hypothesis.

Nevertheless, it is quite possible that some version of the phonological hypothesis can be constructed that correctly predicts the present pattern of results and that is also perceptually plausible. Nearey's logistic framework certainly seems to offer an interesting paradigm for formulating and testing various models. Detailed experimental tests should be carried out both to evaluate new versions of the phonological hypothesis and to seek further corroboration for the integrative routine hypothesis.

An interesting point about the original diphone-biased segmental model with context-sensitive phones is that it implies an exemplar view of phoneme categories. The fact that it does not fit the empirical pattern of results could suggest that phoneme categories are best understood as being represented on the basis of prototypes, an assumption underlying the predictions made for the phonological and integrative routine hypotheses. However, scant research allows a direct empirical evaluation of the exact categorization model underlying phoneme category representations (see, e.g., Medin \& Barsalou, 1987, for discussion of some relevant data), although the fact that phoneme categories generate graded structure is by now well accepted (Decoene, 1993; Kuhl, 1991; Miller \& Volaitis, 1989; Samuel, 1982). Although the results reported here are more compatible with a prototype view than with an exemplar view, additional research on the categorization mechanisms underlying phoneme categories should be undertaken.

The existence of both phoneme category and syllable category representations with perceptual content would allow for a distinct parallel between speech production and perception. If there is a library of (syllable-sized) articulatory routines that details how the (phoneme-sized) constellations of articulatory gestures have to be articulated (as Levelt, 1989, and Levelt \& Wheeldon, 1994, have argued), then the perceptual representation of phoneme and syllable categories actually mirrors the hierarchical relation between core gestures and integrative routines.

Also, the representation of phoneme categories using stable low-level perceptual information and the representation of syllable categories based on higher order perceptual information does not preclude the possibility that the former information base will aid detection of the latter (and vice versa). In fact, the predictions made for the integrative routine hypothesis presupposed precisely such a tradeoff. Also note that the observation that, for example, syllable monitoring is generally faster than phoneme monitoring can be reinterpreted in this way. On this view, faster syllable monitoring is not based on the fact that they are perceptually more basic, but on the fact that syllable targets activate two pieces of informationlow-level perceptual information of phoneme categories and higher order integrative information of syllable categories (e.g., Mills, 1980a, 1980b).

On the basis of the three experiments reported here, several research suggestions can be made. Further experiments should be set up to test directly the degree to which the assumption of phoneme prototypes (instead of phoneme exemplars) can be empirically demonstrated. Another interesting idea is that of stronger coarticulatory coding of VC syllables than of $\mathrm{CV}$ syllables, and its possible representation in syllable categories. Third, the present research explicitly focused on speech sound representation. However, this is only one aspect of speech perception, and the representational hypothesis defended here should be integrated in experiments on speech perception processing and its modeling.

\section{REFERENCES}

ADEs, A. E. (1974). How phonetic is selective adaptation? Experiments on syllable position and vowel environment. Perception \& $P$ sychophysics, 16, 61-66.

Browman, C., \& Goldstein, L. (1986). Towards an articulatory phonology. Phonology Yearbook, 3, 219- 252.

Browman, C., \& Goldstein, L. (1988). Some notes on syllable structure in articulatory phonology. Phonetica, 45, 140-155.

Browman, C., \& Goldstein, L. (1989). Articulatory gestures as phonological units. Phonology Yearbook, 5, 201-251. 
Browman, C., \& Goldstein, L. (1990). Tiers in articulatory phonology, with some implications for casual speech. In J. Kingston \& M. Beckman (Eds.), Papers in laboratory phonology I: Between the grammar and the physics of speech (pp. 341-376). Cambridge: Cambridge University Press.

ClarK, J., \& YalloP, C. (1990). An introduction to phonetics and phonology. Oxford: Blackwell.

COLE, R., \& SCotT, B. (1973). Perception of temporal order in speech: The role of vowel transitions. Canadian Journal of Psychology, 27, 441-449.

Cole, R., \& ScotT, B. (1974). Toward a theory of speech perception. Psychological Review, 81, 348-374.

Collier, R., \& Droste, F. (1982). Fonetiek en Fonologie [Phonetics and phonology]. Leuven: Acco.

DECOENE, S. (1993). Testing the speech unit hypothesis with the primed matching task: Phoneme categories are perceptually basic. Perception \& Psychophysics, 53, 601-616.

DeCOENE, S., \& TIMPERMAN, K. (1996). Lexical effects on primed phoneme and syllable matching. Phoneme categories remain perceptually basic. Manuscript submitted for publication.

Dorman, M. F., CutTing, J. E., \& Raphä̈L, L. J. (1975). Perception of temporal order in vowel sequences with and without formant transitions. Journal of Experimental Psychology: Human Perception \& Performance, 104[1], 121-129.

Elman, J., \& MCClelland, J. (1986). Exploiting lawful variability in the speech wave. In J. Perkell \& D. Klatt (Eds.), Invariance and variability in speech processes (pp. 360-380). Hillsdale, NJ: Erlbaum.

Foss, D., \& Swinney, D. (1973). On the psychological reality of the phoneme: Perception, identification, and consciousness. Journal of Verbal Learning \& Verbal Behavior, 12, 246-257.

HANDEL, S. (1989). Listening: An introduction to the perception of auditory events. Cambridge, MA: MIT Press.

KIRK, R. (1982). Experimental design: Procedures for the behavioral sciences. Belmont, CA: Brooks/Cole.

KUHL, P. K. (1991). Human adults and human infants show a "perceptual magnet effect" for the prototypes of speech categories, monkeys do not. Perception \& Psychophysics, 50, 93-107.

LeVelt, P. (1989). Speaking. From intention to articulation. Cambridge, MA: MIT Press.

LEVELT, P., \& WHEELDON, L. (1994). Do speakers have access to a mental syllabary? Cognition, 50, 239-269.

MASSARO, D. W. (1987). Speech perception by ear and eye: A paradigm for psychological inquiry. Hillsdale, NJ: Erlbaum.

MASsARO, D. W. (1989). Testing between the TRACE model and the fuzzy logical model of speech perception. Cognitive Psychology, 21, 398-421.

Massaro, D. W., \& Cohen, M. C. (1983). Phonological context in speech perception. Perception \& Psychophysics, 34, 338-348.

MASSARo, D. W., \& COHEN, M. C. (1991). Integration and interactive activation: The joint influence of stimulus and context in perception. Cognitive Psychology, 23, 558-614.

MCCLELLAND, J. (1991). Stochastic interactive processes and the effect of context on perception. Cognitive Psychology, 23, 1-44.

MEdin, D., \& BarsaloU, L. (1987). Categorization processes and categorical perception. In. S. Harnad (Ed.), Categorical perception (pp. 455-490). Cambridge: Cambridge University Press.

Miller, J., \& Volaitis, L. E. (1989). Effect of speaking rate on the perceptual structure of a phonetic category. Perception \& Psychophysics, 46, 505-512.

MiLls, C. (1980a). Effects of context on reaction time to phonemes. Journal of Verbal Learning \& Verbal Behavior, 19, 75-83.
MiLls, C. (1980b). Effects of the match between listener expectancies and coarticulatory cues on the perception of speech. Journal of Experimental Psychology: Human Perception \& Performance, 6, 528535.

NEAREY, T. (1990). The segment as a unit of speech perception. Journal of Phonetics, 18, 347-373.

Nearey, T. (1991). Perception: Automatic and cognitive processes. In Proceedings of the XIIth International Congress of Phonetic Sciences (Vol. 1, pp. 40-49). Aix-en-Provence, France: Publications de l'Université de Provence.

NEarey, T. (1992). Context effects in a double-weak theory of speech perception. Language \& Speech, 35, 153-171.

PISONI, D. B., \& LUCE, P. (1987). Acoustic-phonetic representations in word recognition. Cognition, 25, 21-51.

PIsONI, D. B., \& TASH, J. (1975). Auditory property detectors and processing place features in stop consonants. Perception \& Psychophysics, 18, 401-408

Pols, L. (1977). Spectral analysis and identification of Dutch vowels in monosyllabic words. Unpublished doctoral dissertation, Eindhoven: IPO.

PosNer, M. I., \& SNYdER, C. R. R. (1975). Facilitation and inhibition in the processing of signals. In P. M. A. Rabbitt \& S. Dornic (Eds.), Attention and performance V (pp. 669-682). London: Academic Press.

SAMUEL, A. G. (1982). Phonetic prototypes. Perception \& Psychophysics, 31, 307-314.

SAMUEL, A. G. (1989). Insights from a failure of selective adaptation: Syllable-initial and syllable-final consonants are different. Perception \& Psychophysics, 45, 485-493.

SAMUEL, A. G., KAT, D., \& TARTTER, V. (1984). Which syllable does an intervocalic stop belong to? A selective adaptation study. Journal of the Acoustical Society of America, 76, 1652-1663.

SaWusch, J. (1977). Processing of place information in stop consonants. Perception \& Psychophysics, 22, 417-426.

STRANGE, W. (1989). Evolving theories of vowel perception. Journal of the Acoustical Society of America, 85, 2081-2087.

Sussman, H. (1984). A neuronal model for syllable representation. Brain \& Language, 22, 167-177.

Sussman, H. (1986). A neuronal model of vowel normalization and representation. Brain \& Language, 28, 12-23.

Sussman, H. (1989). Neural coding of relational invariance in speech: Human language analogs to the barn owl. Psychological Review, 96, 631-642.

WhaLEN, D. H. (1989). Vowel and consonant judgments are not independent when cued by the same information. Perception \& Psychophysics, 46, 284-292.

WOLF, C. G. (1978). Perceptual invariance for stop consonants in different positions. Perception \& Psychophysics, 24, 315-326.

\section{NOTE}

1. In all three experiments, it was checked whether subjects responded more slowly or with more errors to these stimuli with voiced syllable-final $/ \mathbf{b} /$. This was not the case, showing that subjects did not find these stimuli-which are, strictly speaking, not permissible in Dutch—more difficult than other stimuli.

(Manuscript received September 2, 1994; revision accepted for publication August 7, 1996.) 